\title{
Acknowledgment of Principal and Ad Hoc Reviewers
}

The Editor and Associate Editors also wish to thank the following persons who served as reviewers for Training and Education in Professional Psychology, from September 2016 to September 2017.

Kenneth Adams

Allison Aosved

Victor Barr

Sherry Beaudreau

Rinad Beidas

Deborah Beidel

John Billig

James Boswell

Julie Boydston

Benjamin Buck

David Call

Linda Campbell

Timothy Campellone

Timothy Cavell

Ruth Chu-Lien Chao

David Cimbora

Mary Alice Conroy

Stephen Cook

Stephen Correia

Valerie Crabtree

Christopher Cushing

Edward Dill

Kim Dixon

Halina Dour

Marla Eby

Nabil El-Ghoroury
Timothy Elliott

Michael Ellis

Spencer Evans

Connie Fournier

Miguel Gallardo

Dellanira Garcia

Shirley Glynn

Simon Goldberg

William Gottdiener

Preston Greene

Catherine Grus

Bridget Hegeman

Kimberly Hill

Stephen Holliday

Leah Horvath

Christopher Houck

Jeanette Hsu

John Hunsley

Farah Ibrahim

Sue Jacobs

Rene Jamison

W. Brad Johnson

Justin Karr

Anusha Kassan
Lisa Kearney

W. Gregory Keilin

Sarah Kirk

Linda Knauss

Thad Leffingwell

Keren Lehavot

Steve Lisman

Steve Martino

Bryce McLeod

Mark McMinn

Elizabeth McQuaid

Scott Michael

Rachel Millstein

Nancy Murdock

Douglas Nangle

Britt Nielsen

Sonya Norman

Kati Pagulayan

Janelle Painter

Laura Palmer

David Pantalone

Wendy Paszkiewicz

Margaret Rogers

Joel Schmidt
Richard Seime

Edward Shafranske

Ryan Sharma

Keith Shaw

Paula Shear

Randyl Smith

Steven Smith

Sally Stabb

Ric Steele

William Stiers

Thad Strom

Joshua Swift

Jan Tackett

Karen Tao

Saneya Tawfik

Ray Tucker

Aaron Turner

Jason Van Allen

Kristi Van Sickle

Jared Warren

C. J. Watkins

Rhonda Williams

Michele Willingham

Susan Zlotlow 